\title{
Activated Protein C Resistance: The Most Common Risk Factor for Venous Thromboembolism
}

\author{
Dawn R. Sheppard, DO
}

Background: Venous thromboembolism is a major cause of morbidity and mortality. Although activated protein $\mathrm{C}$ resistance (APC-R) is the most commonly recognized inherited risk factor for venous thromboembolism, little is known about its long-term implications on health.

Metbods: MEDLINE was searched from January 1989 through August 1999 using the key words "thromboembolism," "thrombosis," "activated protein C resistance," and "factor V Leiden."

Results: One in 1000 people in the United States is affected by venous thromboembolism annually. APC-R is now understood to be responsible for up to $64 \%$ of these cases. APC-R, which occurs widely in some ethnic groups and is nearly absent in others, is due to a single point mutation in the gene for clotting factor $V$. As a result, inactivation of factor $V$ by activated protein $C$ is impaired, leading to a hypercoagulable state. This condition creates a lifelong increased risk of thrombosis and, possibly, anticoagulant therapy..

Conclusion: Family physicians have a new tool for assessing risks for venous thromboembolism. Recognizing that up to $64 \%$ of patients with venous thromboembolism can have APC-R and treating this disorder with prophylactic and therapeutic anticoagulation might reduce patient morbidity and mortality from venous thromboembolism. Screening high-risk patients might now be indicated. (J Am Board Fam Pract 2000;13:111-5.)

Venous thromboembolism, a serious health problem and an important cause of morbidity, affects about 1 in 1000 persons annually. ${ }^{1-5}$ It can prolong a hospital stay, it can expose patients to medications and increase their risk of developing an adverse reaction, and it is sometimes fatal. Patients who have spontaneous or recurrent venous thromboembolism could have an underlying inherited or acquired hypercoagulable state. Although patients with venous thromboembolism frequently have family histories of thrombosis, well-defined defects, such as inherited deficiencies of anticoagulant proteins, were found only in a minority (5\% to $10 \%)$ of cases. $^{4-6}$

\section{Methods}

MEDLINE was searched from January 1989 through August 1999 using the search terms

Submitted, revised, 23 September 1999.

From General Leonard Wood Army Community Hospital, Ft Leonard Wood, Mo. Address reprint requests to Dawn R. Sheppard, DO, General Leonard Wood Army Community Hospital, 126 Missouri Ave, Ft Leonard Wood, M0 65473. "thromboembolism," "thrombosis," "activated protein C resistance," and "factor V Leiden."

\section{Background}

Dahlback discovered a novel mechanism for familial thrombosis in 1993. It is characterized by an inherited resistance to the anticoagulant action of activated protein C (APC). ${ }^{2,5}$ Studies report that the discovery of activated protein $C$ resistance (APC-R) has raised the yield of diagnosed coagulation abnormalities up to $64 \%$ in thrombosis patients and up to $8 \%$ in the general population. The wide range is postulated because of differences in selection criteria, ethnic background of the populations studied, and laboratory methods used. ${ }^{1,3,5,7-9}$ APC-R is by far the most common inherited thrombophilia.

\section{Physiology and Pathophysiology}

Within intact vessels, thrombin binds to thrombomodulin on the endothelial cell. This bound thrombin functions as an anticoagulant by activating the protein $\mathrm{C}$ system. APC potentiated by cofactor protein $S$ down-regulates the activity of the 
coagulation system (limits clot formation) by cleaving and inhibiting factors $\mathrm{V}(\mathrm{FV})$ and VIII. ${ }^{2,7,8,10-13}$

APC-R is caused by a single point mutation in the FV gene. ${ }^{2,11}$ This mutated FV may be referred to as factor V Leiden (FVL), FV:Q506 allele, or as APC-R. ${ }^{1,2,13,14}$ This mutated gene is less efficiently degraded by APC (therefore potentiating clot formation). Genotypic carriers of this allele, both heterozygous and homozygous, are at greater risk for venous thromboembolism.

\section{Etiology}

It is not known what positive genetic selection pressure has been involved in maintaining APC- $R$ in the population. ${ }^{2}$ Some experts suggest that the high frequency of FVL allele has conferred a survival advantage during evolution. For instance, a thrombophilic state might have provided protection against severe bleeding episodes in association with postpartum hemorrhage or life-threatening trauma, or it might have protected against iron deficiency by reducing menstrual blood loss., Modern-day life exposes FVL carriers to circumstantial venous thromboembolism risks to which our ancestors were not exposed: oral contraceptives, major surgery, prolonged immobilization during travels, extended life span, and a relatively sedentary lifestyle. . $^{2,7,15,16}$

\section{Epidemiology and Prevalence}

APC-R is the most common inherited risk factor for thrombosis. Many studies report a prevalence of $3 \%$ to $64 \% .^{5,13}$ The likelihood of this disorder occurring is highly dependent on the population studied. Heterozygous FVL occurs in $3 \%$ to $8 \%$ of the general population of white, European descent. ${ }^{7,1,2,4,7,8,15}$ Although the carrier frequency in Hispanic, African-American, Asian, and Native American populations is substantially less, a US study did find FVL in these groups. ${ }^{17}$ Based on ethnic-specific allele frequencies observed in this study and on US census data, it can be estimated that more than 11.3 million Americans carry the mutated FV gene. ${ }^{17}$

\section{Clinical Manifestations}

Venous thrombosis is the third most common cardiovascular disease after acute ischemic heart disease and stroke. ${ }^{8}$ Deep venous thrombosis is the most prevalent manifestation of APC-R, although pulmonary embolism and superficial thrombophlebitis are found in many cases. ${ }^{7,8}$ Rare thrombotic manifestations and arterial thromboses are not commonly reported as in other inherited thrombophilias. ${ }^{7}$ Clinically APC-R occurs with equal risk to both sexes, ${ }^{5,17}$ at any age, and in a variety of manifestations.

FVL is inherited in an autosomal-dominant fashion; but penetrance is highly variable..$^{4,7,8} \mathrm{Al}$ though some never experience thrombotic events, others suffer from recurrent, severe thrombosis at a young age. 1,2,4,7-9,14 In general, homozygous patients experience thrombosis at a much younger age than do heterozygotes (median 31 years compared with 47 years). 9 Thrombotic risk is increased 5 to 10 times more than normal in heterozygotes and 50 to 100 times in homozygotes. ${ }^{1,2,9,15}$ This risk is compounded by the coexistence of other genetic or acquired risk factors. ${ }^{7,8,14}$

Persons with combinations of FVL and another clotting defect have a much higher risk of thrombosis than do those with just one genetic defect $^{2,3,14,18}(72 \%$ and $19 \%$ APC-R only, respectively $\left.{ }^{16}\right)$. Researchers have reported that APC-R is found in up to $19 \%$ of symptomatic protein Cdeficient patients and $39 \%$ of protein S-deficient patients. ${ }^{16}$ The high prevalence of this gene in the population increases the chance that it could coexist with another thrombophilia, such as prothrombin gene mutation, hyperhomocystinemia, antithrombin III deficiency, or antiphospholipid syndrome.

In addition to inherited APC-R, there can be precipitating factors that predispose persons to acquire APC-R. Oral contraceptive intake and pregnancy are associated with an increased prevalence of thrombosis, up to $60 \%$ and $80 \%$, respectively. ${ }^{1,8,13,14,16}$ Women taking oral contraceptives have a much lower APC ratio (defined later in this article) than do matched controls., ${ }^{913}$ Two women labeled with APC-R (without FVL) had their ratios return to normal within 2 months of discontinuing contraceptive medications. ${ }^{9,13}$ The thrombosis risk increases 3.8-fold in those taking oral contraceptives and 35- to 50-fold in those who are FVL carriers and taking oral contraceptives. ${ }^{2,9,17}$ The incidence of venous thromboembolism in young, healthy females is very low, however. If other, less successful contraceptive methods fail, the patient could be at greater risk of venous thromboembolism from an unintended pregnancy. ${ }^{17}$ Currently the literature suggests hor- 
mone replacement therapy might increase venous thromboembolism risk up to twofold, ${ }^{19}$ and hormone replacement therapy combined with APC-R could increase risk up to threefold. ${ }^{20}$ The risk of venous thromboembolism is very small, however, compared with the cardiovascular and other benefits from hormone replacement therapy.

Moreover, $20 \%$ of recurrent miscarriage losses in the second trimester have been associated with FVL. ${ }^{16}$ This information has led some researchers to examine the role of APC-R in conjunction with antiphospholipid syndrome. Anticardiolipin antibodies from some antiphospholipid syndrome patients down-regulate APC activity, in turn creating the thrombophilia associated with antiphospholipid syndrome. ${ }^{21}$ Patients with thrombophilia should be screened for APC-R even if antiphospholipid syndrome has been previously diagnosed. ${ }^{22}$

\section{Differential Diagnoses}

Consideration should be given to ruling out other clinical conditions known to be associated with thrombosis, such as immobilization, obesity, oral contraceptive use, congestive heart failure, pregnancy, infection, malignancy, myeloproliferative disorder, hyperlipidemia, postsurgical state, and nephrotic syndrome. ${ }^{1}$ Attendant circumstantial factors, however, should not preclude an evaluation for inherited thrombophilia. ${ }^{14}$ A precipitating factor is often observed in heterozygous and homozygous persons. ${ }^{14}$ Other coagulation pathway abnormalities that increase the risk for thrombosis include a deficiency of antithrombin III, protein C and protein $S$ deficiencies, abnormalities of the fibrinolytic system (plasminogen, tissue plasminogen activator, and plasminogen activator inhibitor), fibrinogen deficiencies, homocystinemia, prothrombin DNA mutation, and antiphospholipid syndrome. ${ }^{1}$

\section{Laboratory Testing}

Approximately $10 \%$ of all persons afflicted with the APC-R phenotype do not have the APC-R genotype, or FVL. ${ }^{2,14}$ As a result, several different methods are used for screening and confirming APC-R and its mutated FV allele.

\section{APC Test}

The APC test was developed to investigate the anticoagulant response to APC in plasma samples from patients who had unexplained thrombosis. The APC test can be performed on anticoagulated blood. The patient's plasma is prediluted with FVdeficient plasma. ${ }^{9,12}$ (FV-deficient plasma is able to correct the deficiency of the vitamin K-dependent factors without affecting the patient's endogenous $\mathrm{FV}^{9}$ ). A carefully standardized amount of APC is added in an activated partial thromboplastin time (aPTT) reaction. An aPTT test without APC is also performed, and results are expressed as the quotient (APC ratio) of the former clotting time divided by the latter. ${ }^{2,10}$

$$
\begin{aligned}
& {[\mathrm{aPTT}+\mathrm{APC}(\mathrm{sec})] /[\mathrm{aPTT}(\mathrm{sec})]} \\
& =\mathrm{APC} \text { resistance ratio }
\end{aligned}
$$

Patients with APC-R usually have a ratio of $<2.0$ compared with a ratio of $>2.0$ for patients without APC-R. ${ }^{7,10}$ The above procedure is rapid, relatively inexpensive, and highly reproducible if performed under standardized conditions. ${ }^{7}$ The APC-ratio indicates the severity of the APC-R and also detects thrombosis-prone APC-R cases without the FV gene mutation. ${ }^{3}$ The high sensitivity and specificity of the APC ratio (approaching $100 \%^{3,7,8,14}$ ), together with its reliability and ease with which it is performed, make it an adequate screening method. Even so, a certain number of cases fall within a zone that is borderline for diagnosis. ${ }^{3}$ Those who have a borderline test or a positive test can then be analyzed with direct genotyping to confirm the presence of FVL allele. ${ }^{8}$

\section{FV Genotype}

DNA genotyping gives a distinct and certain result: the patient is normal, heterozygous, or homozygous. ${ }^{3}$ Confirming genotype is important to differentiate acquired from inherited APC-R. An inherent disadvantage of proceeding directly to a molecular test is that with approximately $10 \%$ of APC-R (phenotype), patients lack the FV mutation (genotype), ${ }^{1}$ and the diagnosis of APC-R in these patients will be missed. The genotyping technique is not designed to measure function and will not detect other anticoagulant protein deficiencies that stand alone or that add to the severity of APC-R. ${ }^{3}$ The resulting combination of phenotypic and genotypic information will aid in establishing prophylactic and therapeutic guidelines. 


\section{Medical Treatment and Preventive Measures}

Although in patients with APC-R, venous thromboembolism requires much the same treatment and follow-up as it does for in other patients, some management considerations need to be addressed. The prophylactic and therapeutic treatment of APC-R is not well established. The number of sites involved, the severity of the thrombosis, and whether the thrombosis was spontaneous or secondary to a precipitating event ${ }^{1}$ will influence the physician and the patient as they make difficult therapeutic decisions. Family history (frequency of venous thromboembolism among family members and their genotypes), occupational history (risk of trauma), and social history (sedentary lifestyle, reproductive desires) are also important when initiating or maintaining antithrombotic therapy. ${ }^{1,2,8}$

Asymptomatic patients with APC-R who have never had a thromboembolic event, as well as their family members, have special needs. They should receive counseling regarding the implication of the diagnosis and information concerning the signs and symptoms of venous thromboembolism. ${ }^{1}$ Shortterm prophylaxis with heparin must be considered for certain high-risk circumstances known to provoke thrombosis, such as immobilization, surgery, trauma, or obstetric procedures. ${ }^{1,8}$

Heterozygotes, homozygotes, and those with another coagulation defect should be given preventive therapy for circumstantial risks ${ }^{18}$ and extended anticoagulation after a thrombotic event. ${ }^{8}$ The physician and the patient must balance the risk of bleeding against the risk of recurrence when therapy is discontinued. Retrospective data have shown a long period between treatment interruption and recurrence (mean 10 years). ${ }^{14}$ Even so, many physicians empirically treat patients for at least 1 year after two episodes of venous thromboembolism and prescribe life-long treatment after three episodes. ${ }^{23}$ Further studies are needed to define the optimal type, intensity, and duration of prophylactic and therapeutic options.

\section{Screening and Future Direction}

Patients with venous thromboembolism should be screened for an underlying coagulation abnormality (including, but not limited to APC-R) when any of the following conditions are encountered: family history of thrombosis; thrombosis at an early age ( $<45$ years); recurrent thrombosis, especially with- out obvious precipitating factors; recurrent thrombosis despite adequate anticoagulant therapy; and thrombosis at an unusual site (such as the hepatic, mesentery, or axillary vein or sagittal sinus). ${ }^{1,16,23}$ Screening for an inherited thrombophilia (including, but not limited to APC-R) is also indicated for any woman who experiences a deep vein thrombosis during pregnancy or oral contraceptive use, and screening must be considered in managing recurrent fetal losses.

In the past, general screening for inherited thrombophilia before circumstances warranted has not been judged to be cost-effective, in part because clotting defects are uncommon. The clear association between APC-R and increased risk of thrombosis, together with a $3 \%$ to $64 \%$ prevalence of $A P C-R$ in the general population, mandate the need for more prospective data. These data will help determine the benefits of general screening in association with surgery, oral contraceptive use, pregnancy, and other high-risk situations. ${ }^{2,3,7}$ It will provide important information to family members and help determine management options. For example, should women have APC-R screening in association with oral contraceptive use? If a woman is found to be phenotype positive and genotype negative while taking oral contraceptives, she might elect to choose another contraceptive method, thus potentially avoiding a venous thromboembolism or long-term anticoagulation. Questions such as these will need to be answered.

\section{Conclusion}

The efficacy, safety, and need for APC-R screening of high-risk patients might now be indicated. The widely ranging prevalence of APC-R and the availability of laboratory testing will have a profound influence on the development of therapeutic and prophylactic regimens. A number of unresolved issues must be addressed, however, before widespread screening can be implemented. Clinical trials are needed to determine the optimal anticoagulation needed to reduce morbidity and mortality from APC-R-induced venous thromboembolism. Our understanding of APC-R is advancing, but testing for APC-R is ordered far less frequently than for other inherited thrombophilia, ${ }^{16}$ which suggest routine testing might not be available or practicing clinicians are unaware of this entity. Educating ourselves, our patients, and our fellow phy- 
sicians about this most common thrombophilic disorder is of utmost importance.

\section{References}

1. Bridgen ML. The hypercoagulable state. Who, how, and when to test and treat. Postgrad Med 1997;101: 249-52, 254-62, passim.

2. Dahlback B. New molecular insights into the genetics of thrombophilia. Resistance to activated protein $\mathrm{C}$ caused by $\operatorname{Arg} 506$ to GLn mutation in factor $\mathrm{V}$ as a pathogenic risk factor for venous thrombosis. Thromb Haemost 1995;74:139-48.

3. Dahlback B. Resistance to activated protein $C$, the Arg506 to Gln mutation in the factor $V$ gene, and venous thrombosis. Functional tests and DNA-based assays, pros and cons. Thromb Haemost 1995;73: 739-42.

4. Dahlback B. Physiological anticoagulation. Resistance to activated protein $\mathrm{C}$ and venous thromboembolism. J Clin Invest 1994;94:923-7.

5. Hainaut P, Azerad MA, Lehmann E, et al. Prevalence of activated protein $C$ resistance and analysis of clinical profile in thromboembolic patients. A Belgian prospective study. J Intern Med 1997;241:42733.

6. Dahlback B, Carlsson M, Svensson P. Familial thrombophilia due to a previously unrecognized mechanism characterized by poor anticoagulant response to activated protein C: prediction of a cofactor to activated protein C. Proc Natl Acad Sci USA 1993;90:1004-8.

7. Zoller B, Hillarp A, Berntorp E, Dahlback B. Activated protein $C$ resistance due to a common factor $V$ gene mutation is a major risk factor for venous thrombosis. Annu Rev Med 1997;48:45-58.

8. Hillarp A, Zoller B, Dahlback B. Activated protein C resistance as a basis for venous thrombosis. Am J Med 1996;101:534-40.

9. Chrobak L, Dulicek P. Resistance to activated protein $C$ as pathogenic factor of venous thromboembolism. Acta Medica (Hradec Kralove)1996;39:5562.
10. Svensson PJ, Dahlback B. Resistance to activated protein $\mathrm{C}$ as a basis for venous thrombosis. $\mathrm{N}$ Engl J Med 1994;330:517-22.

11. Dahlback B. Factor $V$ And protein $S$ as cofactors to activated protein C. Haematologica 1997;82:91-5.

12. Bertina RM, Koeleman BP, Koster T, et al. Mutation in blood coagulation factor $\mathrm{V}$ associated with resistance to activated protein C. Nature 1994;369: 64-7.

13. Harris JM, Abramson N. Evaluation of recurrent thrombosis and hypercoagulability. Am Fam Physician 1997;56:1591-6, 1601-2.

14. Samama MM, Simon D, Horellou MH, Trossaert M, Elalamy I, Conard J. Diagnosis and clinical characteristics of inherited activated protein $\mathrm{C}$ resistance. Haemostasis 1996;26:315-30.

15. Rees D, Cox M, Clegg JB. World distribution of factor V Leiden. Lancet 1995;346:1133-4.

16. Florell SR, Rodgers GM. inherited thrombotic disorders: an update. Am J Hematol 1997;54:53-60.

17. Ridker PM, Miletich JP, Hennekens $\mathrm{CH}$, Buring JE. Ethnic distribution of factor V Leiden in 4047 men and women. JAMA 1997;277:1305-7

18. Girolami A, Simioni P, Scarano L, Girolami B. Venous and arterial thrombophilia. Haematologica 1997;82:96-100.

19. Levesque $H$, Courtois $H$. [Estrogen therapy and venous thromboembolic disease]. Rev Med Interne 1997;18(Suppl 6):S620-5.

20. Barlow DH. HRT and the risk of deep vein thrombosis. Int J Gynaecol Obstet 1997;59(Suppl 1):S29_ 33.

21. Griffin JH, Heeb MJ, Kojìma Y, et al. Activated protein $\mathrm{C}$ resistance: molecular mechanisms. Thromb Haemost 1995;74:1:444-8.

22. Ruiz-Arguelles GJ, Garces-Eisele J, Alarcon-Segovia D, Ruiz-Arguelles A. Activated protein C resistance phenotype and genotype in patients with primary antiphospholipid syndrome. Blood Coagul Fibrinolyis 1996;7:344-8.

23. Ginsberg JS. Management of venous thromboembolism. N Engl J Med 1996;335:1816-28. 